\title{
Promoter activity of genes encoding the Specific Tissue protein family in the reproductive organs of Medicago truncatula
}

\author{
L. ALBORNOS, I. MARTÍN, E. LABRADOR*, and B. DOPICO \\ Departamento de Botánica y Fisiología Vegetal, Centro Hispano Luso de Investigaciones Agrarias, \\ Universidad de Salamanca, Salamanca E-37007, Spain
}

\begin{abstract}
The "Specific Tissue" (ST) are proteins of unknown function present only in some plant families, mainly Fabaceae and Asteraceae. They are included in the PF10950 protein family and characterized by the presence of at least one domain of unknown function (DUF)2775. In this work we studied the involvement of the six members of the Medicago truncatula ST family (ST1 to ST6) in the development of flowers, fruits, and seeds by analysing the activity of their promoters ( $p$ ST) after the construction of $M$. truncatula transgenic plants expressing the $\beta$-glucuronidase (GUS) reporter gene under the control of the six $p S T s$. The GUS activity was analysed in whole flowers and fruits and also in histological sections of these organs. The $p S T$ expression in the reproductive organs was mainly associated with the vascular bundles, especially throughout fruit development. These results pointed to an important role of ST proteins during the reproductive development stage, related to nutrient mobilization during the fruit and seed formation, that could be facilitated by their presence in the pod vascular bundles, as well as in the connective tissue of the anthers (ST3, ST4, ST6), in the placenta, the funiculus, and the outer parts of the developing seed (ST2, ST3, ST6). The observations made in this study were in agreement with the functions previously established for the three groups of $M$. truncatula ST proteins, as in the proposed function for ST1 in the transport and assimilation of nutrients, or the involvement of ST4, ST5, and ST6 in floral defence.
\end{abstract}

Additional key words: barrel medic, DUF2775, flowers, fruits, nutrient mobilisation, vascular bundles.

\section{Introduction}

The Specific Tissue proteins (STs) are proteins present in specific plant families, mainly Fabaceae and Asteraceae (Albornos et al. 2012a). These proteins have a signal peptide, an intermediate region with conserved characteristics and several tandems repeats of 25/26 amino acids. The main feature of STs is the presence of at least one DUF2775 domain (PF10950) (Albornos et al. 2012a).

The ST proteins have been related, according to their gene expression profile, to biotic interactions as symbiosis (Liu et al. 2007, Gaude et al. 2012) and to responses to abiotic stresses (Muñoz et al. 1997, Hernández-Nistal et al. 2010, Albornos et al. 2017), although their function remains unknown. Some STs have been associated with developmental processes such as early fruit morphogenesis (Fernández et al. 2007, Wechter et al. 2008), cell elongation (De Vries et al. 1985, Muñoz et al. 1997) or germination (Hernández-Nistal et al. 2006). Also, a role as storage proteins or their relationship with changes in the plant nutritional status, have been proposed (De Vries et al. 1985, Albornos et al. 2014, 2017).

The ST proteins are encoded by multigenic families, the biggest one with six members in barrel medic (Medicago truncatula). Thus the M. truncatula ST gene family has gained interest as a study model. Each member of the family might have a specific role, and hence their expression would be differentially regulated throughout development and/or under diverse stimuli, as it has been previously confirmed (Albornos et al. 2017, 2018).

Submitted 6 March 2019, last revision 11 July 2019, accepted 2 September 2019.

Abbreviations: CRE - cis-acting regulatory element; eGFP - enhanced green fluorescent protein; DUF - domain of unknown function; F1 - developing flower which appears green and closed; F2 - developing flowers with sepals and petals, yellow and closed; F3 fully developed flower at/or after anthesis; F4 - flower after anthesis showing a curved carpel and elongated style; FB - floral bud; Fr1 - developing fruit showing one or more complete spirals, with no spines and not stacked; Fr2 - developing fruit showing barrel morphology with spines, still not hardened; Fr3 - developing fruit showing barrel morphology with spines, already hardened; GUS $\beta$-glucuronidase; $p S T$ - ST promoter; ST- specific tissue; X-GlcA - 5-bromo-4-chloro-3-indolyl $\beta$-D-glucuronic acid.

Acknowledgements: This work was funded by the Spanish Ministry of Science and Technology (BFU2013-44793-P). Lucía Albornos was supported by a Training of University Teachers Grant (FPU) from the Spanish Ministry of Education, Culture and Sport. We also thank Dr. Barker and Dr. Chabaud from the LIPM in Toulouse (CNRS-INRA), for training Lucía Albornos in the Medicago truncatula transformation protocol.

* Corresponding author; e-mail: labrador@usal.es 
All the ST proteins enter the secretory pathway owing to the above-mentioned presence of a signal peptide, and most of them are possibly targeted to the extracellular compartment (Albornos et al. 2012a). Regarding the M. truncatula ST family, it has been established that all the six members are located in the cell wall, although ST2, ST3, and ST6 have a double location both in the cell wall and in the cytoplasm (Albornos et al. 2017, 2018).

In previous reports we have separated the M. truncatula ST proteins in three functional groups (Albornos et al. 2017, 2018). Thus, ST1, which is the only protein included in the first group, might participate in processes affected by nutritional status; the second group integrates ST2 and ST3, that seem to act when the availability of water is limited, not only due to environmental factors but also in physiological controlled desiccation processes such as the seed maturation (Albornos et al. 2017), while ST4, ST5, and ST6 (third group) might be involved in specific biotic interactions through different signalling pathways (Albornos et al. 2018) being their RNAs specifically and differentially up-regulated by methyl jasmonate (MeJA), ethylen (ET), and salicylic acid (SA). However, the specific functions of each ST protein are not completely established yet, and some results indicated discrepancies between the amount of ST transcripts in M. truncatula and the activity of the ST promoters $(p S T)$ when they were expressed in Arabidopsis thaliana, mainly during its reproductive phase (Albornos et al. 2017, 2018).

An involvement of the ST proteins in the development of flowers, fruits, and seeds has been reported in different species (Williams et al. 1990, Fernández et al. 2007). Also, in $M$. truncatula, it has been described that all the $p S T$ present some cis-acting regulatory elements (CREs) associated with the reproductive development and in vivo, the pSTs in Arabidopsis transgenic plants become transiently active at some reproductive structures (Albornos et al. 2017, 2018). This indicated a different function for each ST in these organs and leaded us to deepen the study.

Although the activity pattern of several plant promoters is conserved even between angiosperms and gymnosperms (Germain et al. 2012) indicating the universal functionality of CREs, differential performance has also been reported (Terzaghi and Cashmore 1995, Hernandez-Garcia and Finer 2014). Thus, taking into account the different types of structure and development of flowers and fruits between $A$. thaliana and M. truncatula, together with the above-mentioned discrepancies, we have considered the necessity to study the activity of the $p S T s$ in transgenic plants of $M$. truncatula. Thus, the aim of this work was to construct $M$. truncatula transgenic plants expressing the $\beta$-glucuronidase reporter gene (GUS) under the control of the different $p S T s$, and to study their activity during reproductive development. In order to have a clearer picture of this activity and considering that the specificity of pST activation could be manifested at the tissue level, we have analysed not only the GUS activity in whole flowers and fruits but also within the tissues after making histological sections of these organs.

\section{Materials and methods}

Plants and growth conditions: Medicago truncatula Gaernt. ecotype Jemalong line 2HA seeds were chemically scarified with $95 \%$ (v/v) $\mathrm{H}_{2} \mathrm{SO}_{4}$, surface sterilized with $5 \%(\mathrm{~m} / \mathrm{v})$ chlorine solution for $2 \mathrm{~min}$, placed in Petri dishes with modified Fahräeus medium supplemented with $\mathrm{NH}_{4} \mathrm{NO}_{3}$ to a final $1 \mathrm{mM} \mathrm{N}$ concentration (Fahräeus-N) (Barker et al. 2006), and stratified at $4{ }^{\circ} \mathrm{C}$ in the dark for $2 \mathrm{~d}$. Seeds were germinated in the dark at $25{ }^{\circ} \mathrm{C}$ for 16 to $24 \mathrm{~h}$.

To obtain leaf explants for genetic transformation, 4 germinated seeds were planted in Magenta boxes containing SHb10 medium (Schenk and Hildebrandt 1972) and incubated for 3 weeks in a growth chamber (Aralab, Lisbon, Portugal) at a temperature of $25{ }^{\circ} \mathrm{C}$, a $16-\mathrm{h}$ photoperiod, and an irradiance of $200-300 \mu \mathrm{mol} \mathrm{m} \mathrm{m}^{-2} \mathrm{~s}^{-1}$ (provided by a mix of cool-white and red fluorescent tubes).

For growing plants in pots, germinated seeds were transferred to a mixture of soil:Vermiculite (3:1), and grown in the chamber described above for $15 \mathrm{~d}$ and afterwards transferred to the greenhouse. Flowers and fruits at different developmental stages were collected from plants growing in the greenhouse and immediately submerged in $90 \%$ cold acetone in order to perform GUS staining (see below). The stages of flowers and fruits analysed were: floral bud (FB); flower 1 (F1): the sepals cover the developing flower which appears green and closed; flower 2 (F2): developing flowers with sepals and petals, yellow and closed; flower 3 (F3): fully developed flower at/or after anthesis; flower 4 (F4): flower after anthesis showing a curved carpel and elongated style; fruit 1 (Fr1): developing fruit showing one or more complete spirals, with no spines and not stacked; fruit 2 (Fr2): developing fruit showing barrel morphology with spines, still not hardened and fruit 3 (Fr3): developing fruit showing barrel morphology with spines, already hardened.

Medicago truncatula pST::eGFP-GUS transgenic plants: The six $p S T s$ were cloned into pKGWFS7 destiny vector as indicated in Albornos et al. (2017, 2018), using Gateway $^{\mathrm{TM}}$ cloning technology to construct the $p S T:: e G F P$ GUS transgenes. Binary vectors were introduced into Agrobacterium tumefaciens hypervirulent strain AGL1 by electroporation. $M$. truncatula $\mathrm{cv}$. Jemalong $2 \mathrm{HA}$ was transformed and regenerated via somatic embryogenesis as described in Chabaud et al. (2003). Briefly, wounded leaf explants were cocultured for $3 \mathrm{~d}$ with the agrobacteria, decontaminated, and subsequently transferred to callus inducing medium and embryo inducing medium with kanamycin selection. Every callus developed in the selection media was isolated so the embryos arising from each one come from independent transformation events. The somatic embryos generated were committed to form stems and leaves and, later roots. Once the plantlets developed in vitro a good root system they were acclimated in pots and transferred to the greenhouse (generation T1) until the seeds (generation T2) could be collected.

Prior to the acclimation, regenerated plants were 
checked for the presence of the transgene. To obtain gDNA, one trifolium was collected, immediately frozen in liquid $\mathrm{N}$ and ground using a MM 400 Mixer mill (Retsch, Haan, Germany). The powder was used for gDNA extraction by the CTAB method as described in Doyle and Doyle (1987) using $0.5 \mathrm{~cm}^{3}$ of extraction buffer [2\% $(\mathrm{m} / \mathrm{v}) \mathrm{CTAB}$, $100 \mathrm{mM}$ Tris- $\mathrm{HCl}$ (pH 8.0), 1.4 M NaCl, 20 mM EDTA]. The pellet was resuspended in $0.05 \mathrm{~cm}^{3}$ of MilliQ sterile water and $2 \mathrm{~mm}^{3}$ were used for the PCR using the primer forward 5'-CATCGGCGGGGGTCATAACG-3' that annealed in the kanamycin gene and the primer reverse 5'-TGTGGTCGGGGTAGCGGCTG-3' that annealed in the enhancer green fluorescent protein $(e G F P)$ using the Kappa Taq DNA polymerase (Kappa Biosystems, Wilmington, MA, USA).

Staining GUS: $M$. truncatula transgenic plants bearing the construction $p S T:: e G F P-G U S$ were used to study the promoter activity by histochemical localization of GUS enzymatic activity that produce blue colour when hydrolysing the 5-bromo-4-chloro-3-indolyl- $\beta$ D-glucuronic acid (X-GlcA) (Duchefa, Haarlem, The Netherlands) substrate performed as described by Albornos et al. (2012b).

Plant material was incubated overnight at $37^{\circ} \mathrm{C}$ in GUS staining solution containing $1 \mathrm{mM} \mathrm{X-GlcA}, 50 \mathrm{mM} \mathrm{Na}$ phosphate buffer, $\mathrm{pH} 7.0,2 \mathrm{mM}$ potassium ferrocyanide, $2 \mathrm{mM}$ potassium ferricyanide and $0.2 \%(\mathrm{v} / \mathrm{v})$ Triton $X-100$ and GUS-stained tissues were cleared in $70 \%$ (v/v) ethanol. Activity of GUS was assayed in wild-type (WT) and transgenic plants in flowers and fruits along its development from adult plants growing in the greenhouse.
Images were acquired using a Leica M205 FA stereomicroscope equipped with a Leica DFC495 camera (Leica Microsystems, Mannheim, Germany).

Experiments were performed in at least three plants of three independent lines. Only when several independent transgenic lines displayed the same pattern of expression of the reporter gene was it consider as positive. Wild type control plants showed no GUS activity (data not shown).

Histological analysis: After performing the GUS staining as described above, flowers and fruits were fixed in FAA solution (formalin : acetic acid : $50 \%$ ethanol, 5:5:90) during $72 \mathrm{~h}$. Samples were then dehydrated in increasing concentrations of ethanol and xylene/ethanol and embedded in paraffin (Paraplast Plus, Sigma, St. Louis, USA) embedding medium at $60^{\circ} \mathrm{C}$. The embedded tissues were sliced into $12 \mu \mathrm{m}$ sections using a microtome (HM310, Microm, Walldorf, Germany) and fixed to slides with $0.04 \%(\mathrm{~m} / \mathrm{v})$ poly-lysine. Sections were deparaffinized with xylene and mounted in Entellan (Merck, Darmstadt, Germany). Photographs were taken using a microscope Leica DM 4000 LED equipped with a digital camera Leica DFC550 and using LAS v. 4.3 software (Leica Microsystems).

\section{Results}

Medicago truncatula transgenic plants pST::GUS carrying the $p S T:: e G F P-G U S$ transgene (hereafter $p S T:: G U S$ ) were generated to determine the activity of these promoters. They were obtained as indicated in Materials and methods,

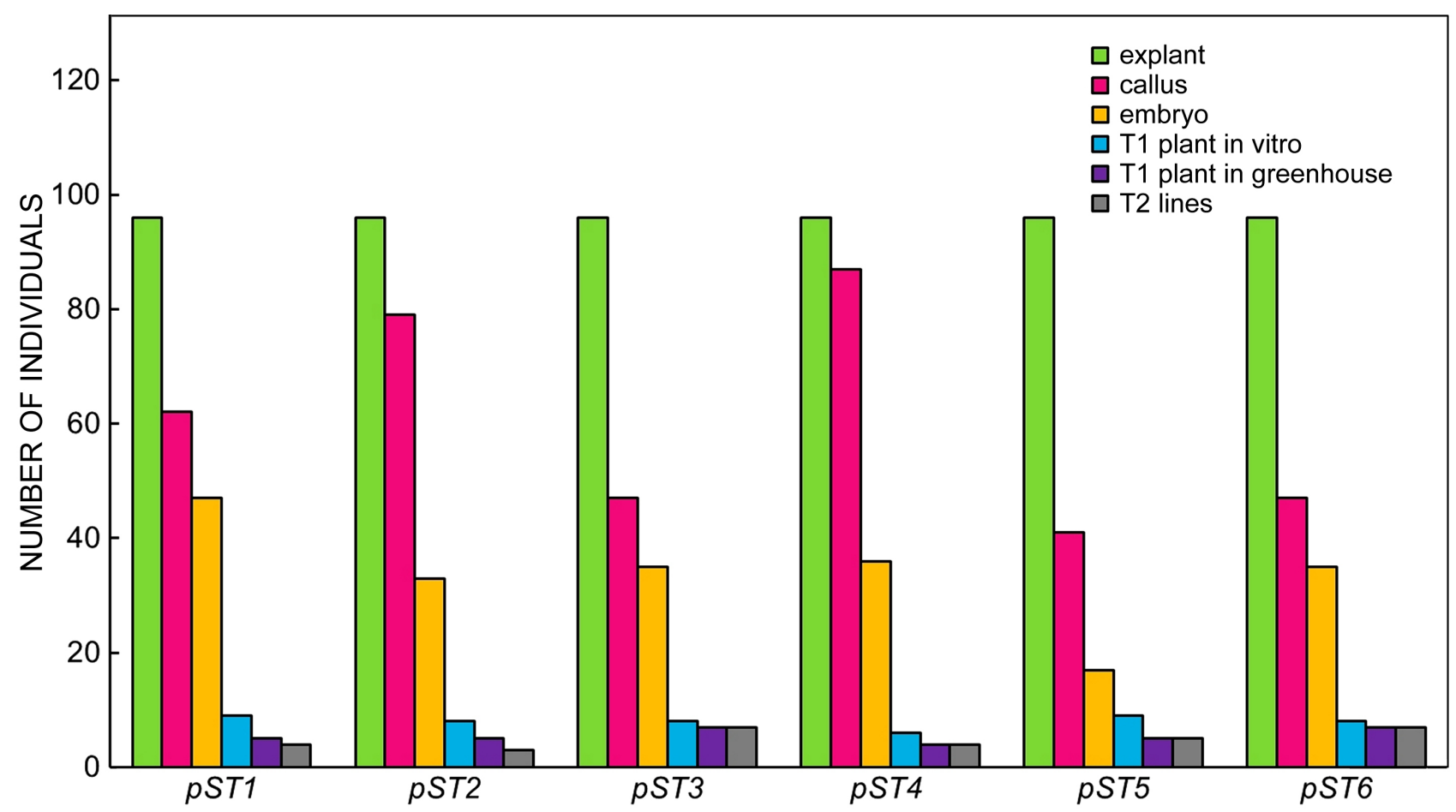

Fig. 1. The percentage of individuals that survived in each phase of the regeneration of the transgenic plants. 

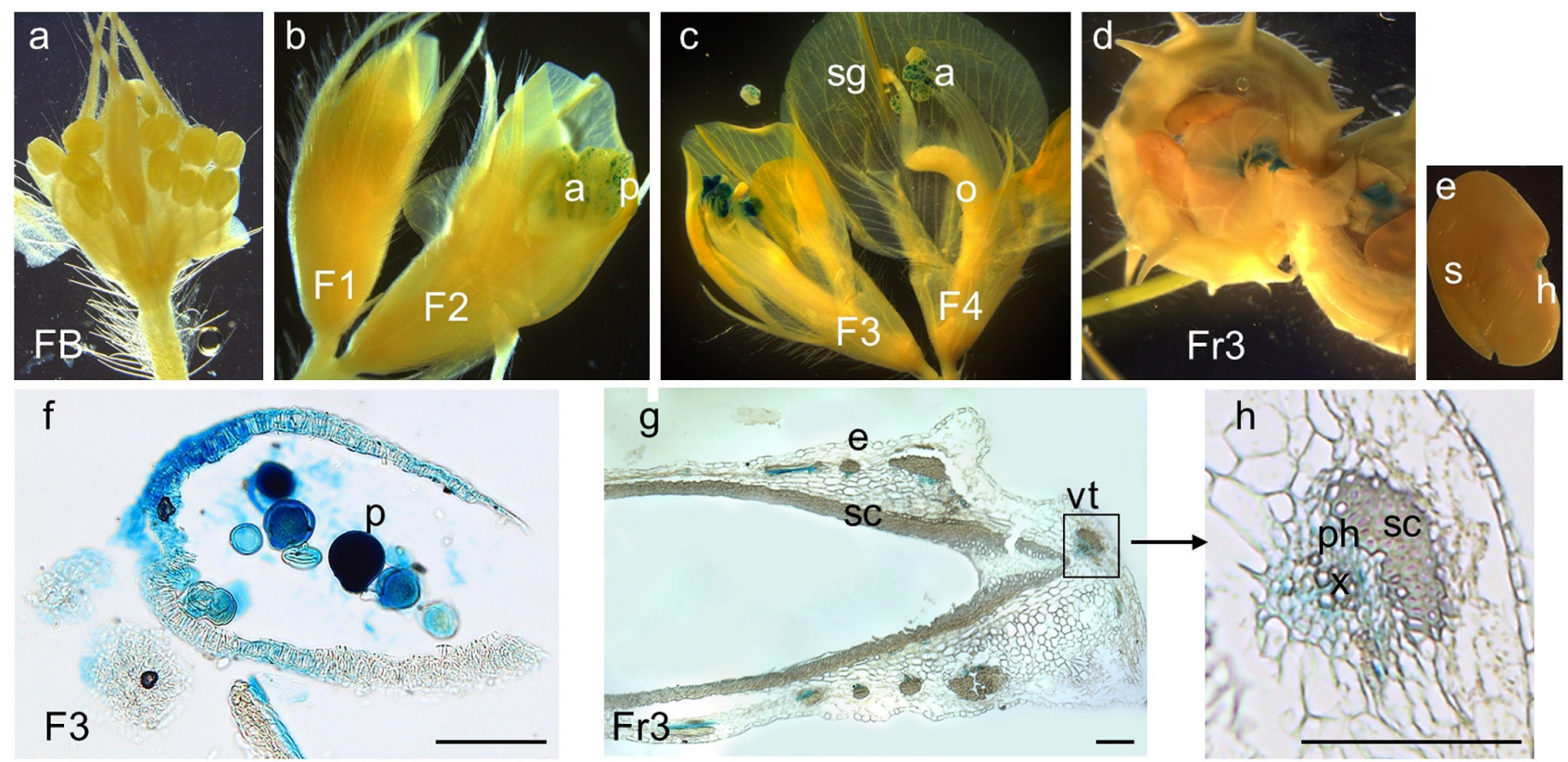

Fig. 2. Activity of Medicago truncatula pST1 in reproductive organs. Histochemical $\beta$-glucuronidase (GUS) staining during reproductive development of $M$. truncatula pST1::GUS plants, sorted according to developmental stage $(A-E)$ and histological sections after GUS staining $(F-H)$. $A$ - floral bud, FB, $B$ - developing flowers F1 and F2, $C$ - fully developed flower F3 and flower after anthesis F4, $D$ - green pod Fr3, $E$ - developing seed in Fr3, $F$ - F3 anther section, $G$ - Fr3 pod section, $H$ - Fr3 pod section detail. The blue colour indicates the zones with GUS activity driven by $p S T 1$; a - anther, h - hilum, o - ovary, $\mathrm{p}$ - pollen, ph - phloem, s - seed, sc - sclerenchyma, sg - stigma, $\mathrm{v}$ - vascular tissue, $\mathrm{x}$ - xylem; scale bars $=100 \mu \mathrm{m}$.

using the AGL1 strain of $A$. tumefaciens. The main difficulty was their regeneration from the cells transformed after the co-cultivation with the agrobacteria (Fig. 1). The limiting step in the regeneration was to obtain plants from somatic embryos. Only between 14 and $19 \%$ of the embryos were able to develop plants, of which $55-87 \%$ survived. At the end of the process, the regeneration rates achieved ranged between $3.1 \%(p S T 2)$ and $7.3 \%(p S T 3$ and $p S T 6$ ), considering only those plants that produced seeds once acclimatized (called T2 lines).

Before the acclimation of the plants, it was verified by PCR that they had incorporated the transgene, acclimatizing only those plants. Non-transformed regenerated plantlets were rarely found (data not shown). The transgenic plants that survived the acclimatization (generation T1) were grown in the greenhouse until they produced seeds (generation T2).

The $M$. truncatula pST::GUS transgenic plants were used to study the activity of the promoters in different tissues and stages of flowers and fruits during the reproductive development by locating the GUS activity with the substrate X-GlcA that produces blue stain. To facilitate the visualization of the activity profiles of each $p S T$, the photographs of the transgenic lines with more intense colour were selected, being shown, in most cases, only those organs in which GUS activity was observed. Since the specificity of activation of $p S T$ could occur at the tissue level, histological sections from flowers and fruits were also made once the enzymatic reaction of GUS was carried out.

The activity of $p S T 1$ was specifically observed in pollen. While the staining could not be detected in floral buds (FB) with the stamen shorter than the carpel (Fig. 2a), it was observed in the pollen grains from closed flowers (F2) with well-developed petals (Fig. 2b); the pollen labelling remained after fertilization when the fruit began to develop (F4) (Fig. 2c). Once the pod reached the characteristic barrel morphology (Fr3), the labelling could be detected in the funiculus and in the seed hilum (Fig. $2 d-e$ ). Histological sections of anthers of F3 clearly showed GUS staining not only in pollen grains but also in the epidermis of the anthers (Fig. 2f). Pod sections revealed pST1 activity in the vascular bundles of Fr3 (Fig. $2 g, h$ ), which could not be noted when the whole fruits were observed.

During the development of the flowers, the $p S T 2$ was only activated in the stamens (Fig. $3 a$ ) being the blue colour specifically located in the apical zone of the filaments and especially in the place where they join the anther but not in the pollen grains (Fig. $3 b$ ). This activity remains in the anthers after the fertilization, when the carpel has given rise to the pod (Fig. $3 c$ ). During the early stages of fruit formation (Fr1), pST2 activity was also found in the peduncle (Fig. $3 c$ ) as well as in the developing seeds (Fig. 3d). In more advanced stages of the fruit development (Fr3), when the pod presents thorns and begins to harden, blue staining was maintained in the join between the fruit and the peduncle (Fig. $3 e$ ) and was also found in the external vascular bundle, the median carpellary bundle (Fig. 3f), but not in the inner ones (Fig. 3g). Histological sections of anthers show that blue staining is limited to the internal layers of the connective tissue (Fig. 3h). Regarding 

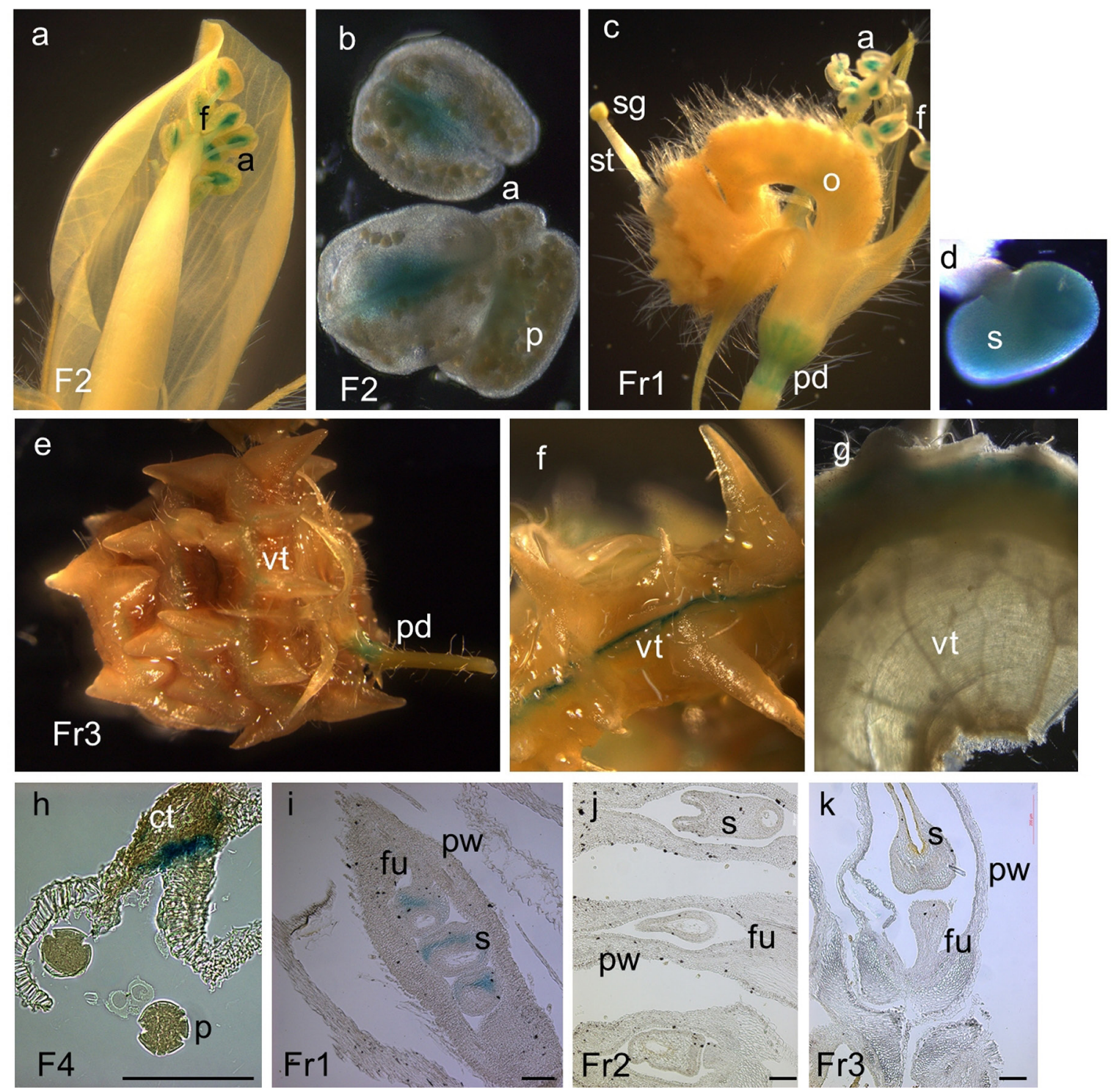

Fig. 3. Activity of Medicago truncatula pST2 in reproductive organs. Histochemical $\beta$-glucuronidase (GUS) staining during reproductive development of $M$. truncatula pST2::GUS plants, sorted according to developmental stage $(A-G)$ and histological sections after GUS staining $(H-K) . A$ - Developing flower F2, $B$ - F2 anthers $C$ - developing fruit Fr1, $D$ - developing seed in Fr1, $E$ - green pod Fr3, $F, G$ Fr3 pod detail, $I$ - developing fruit Fr1 and seeds section, $J$ - Fr2 pod and seeds section, $K$ - Fr2 pod and seeds section. The blue colour indicates the zones with GUS activity driven by $p S T$; a - anther, ct - connective tissue, $\mathrm{f}$ - filament, fu - funiculus, o - ovary, $\mathrm{p}$ - pollen, p - peduncle, pw - pod wall, s - seed, sg - stigma, st - style,; vt - vascular tissue; scale bars $=100 \mu \mathrm{m}$.

the developing fruit and seed sections, the blue labelling was exclusively found in the seeds at the beginning of fruit development, Fr1 (Fig. 3i); this staining could not be appreciated in histological sections from more developed pods, Fr2 and Fr3 (Fig. $3 \mathrm{j}$ and $k$, respectively). pST2 was neither active in the tissues of the pod wall nor in the funiculus throughout fruit development (Fig. $3 i, j, k$ ).

During flower formation, the pST3 was activated in pollen and anthers. A clear staining was observed in the anthers of F2 and F3 when the petals and the carpel were fully formed and the style was not folded (Fig. 4a). The staining began to disappear shortly after fertilization (F4) when the carpel begins to elongate to form the fruit (Fig. $4 b, c)$. The $p S T 3$ activity could be appreciated in the seeds from the beginning of their development (F4) until the fruit reached its definitive morphology (Fr2) (Fig. 4c-e) and showed no staining at the final stage of their formation (Fr3) (Fig. 4f,g). Pods appeared stained throughout its development, from Fr1 to Fr3 (Fig. 4d-f), with the GUS activity located on the placenta (box in the Fig. 4d).

The F3 anther sections confirmed pST3 activity in pollen and especially in the connective tissue (Fig. 4h). The histological analysis of the pods at different stages of development (Fig. 4i-k) showed the GUS activity associated to seeds from early development phases (Fr1) in funiculus, raphe, and chalaza. In Fr1 blue staining was strong in the placenta (Fig. 4j) and extended from the funiculus through the integuments region, and in Fr3 

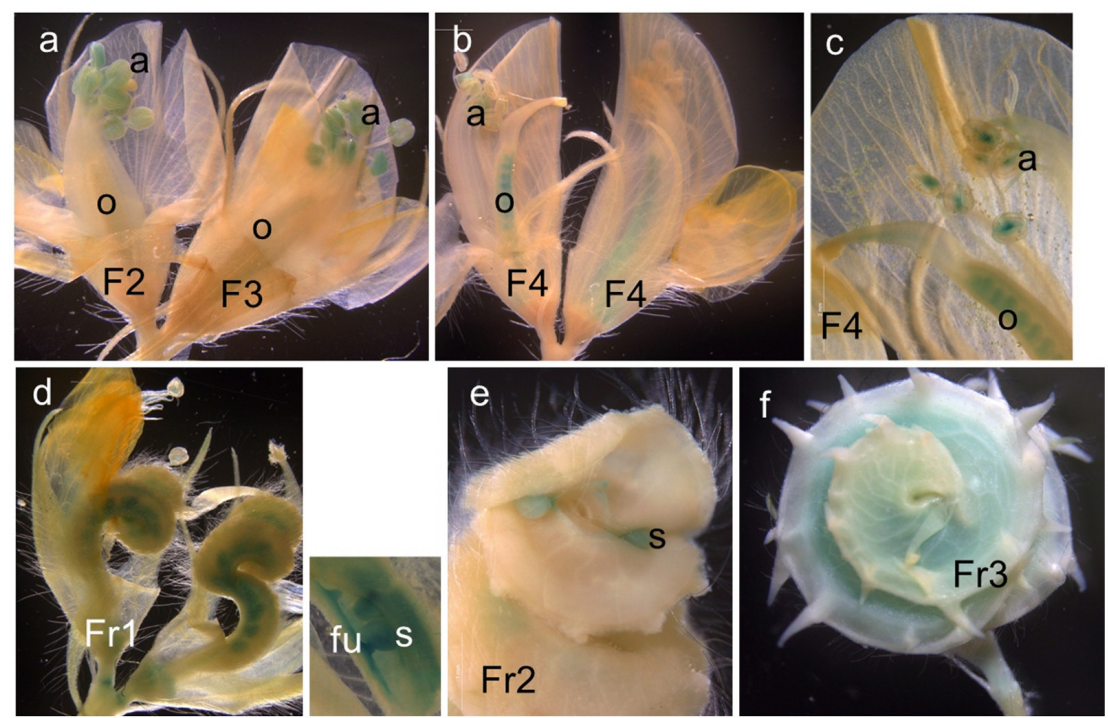

$\mathrm{h}$
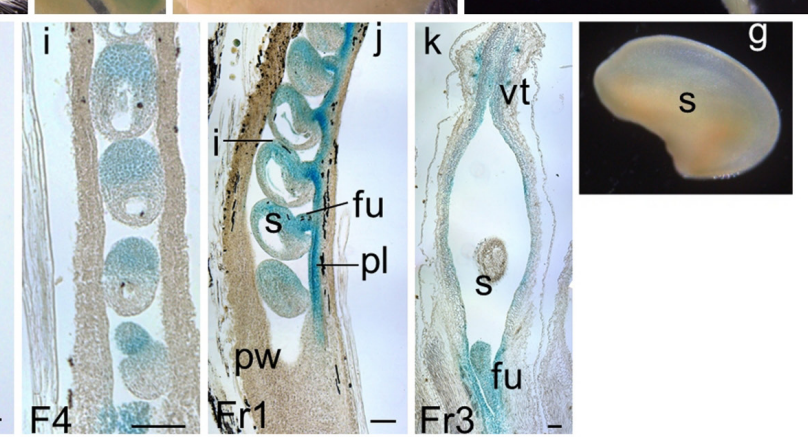

F3

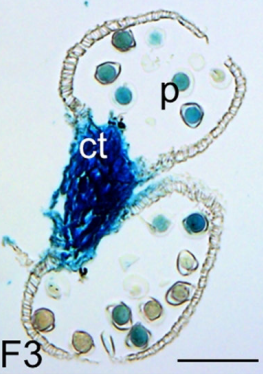

F4 Fir1

Fig. 4. Activity of Medicago truncatula pST3 in reproductive organs. Histochemical $\beta$-glucuronidase (GUS) staining during reproductive development of $M$. truncatula pST3::GUS plants, sorted according to developmental stage $(A-G)$ and histological sections after GUS staining $(H-K)$. $A$ - Developing flower F2 and fully developed flower F3, $B, C$ - mature flower F4 after anthesis, $D$ - developing fruit Fr1, $E$ - developing seeds inside the fruit Fr2, $F$ - green pod $\mathrm{Fr} 3, G$ - developing seed in Fr3, $H$ - anther section in F3, $I$ - early developing fruit section in F4, $J$ - developing fruit Fr1 section, $K$ - green pod Fr3 section. The blue colour indicates the zones with GUS activity driven by pST3; a - anther, ct - connective tissue, fu - funiculus, i - integuments, o - ovary, $\mathrm{p}$ - pollen, pl - placenta, pw - pod wall, s - seed, $\mathrm{vt}$ - vascular tissue; scale bars $=100 \mu \mathrm{m}$.

the activity was shown in vascular bundles, endocarp, and also in funiculus (Fig. $4 k$ ). It should be noted that funiculus activity was maintained in Fr3 but seed activity disappeared (Fig. 4k).

The pST4 was the only one showing activity in FB (Fig. 5a) and this activity decreased rapidly as the development progressed. Besides, in F2 the promoter was activated in anthers and filaments (Fig. $5 b$ ) and, once fertilization occurred, fruit showed GUS staining, first in the apical zone and later all over the organ (Fig. $5 c-f$ ). pST4 was also active in the funiculus, but not in the developing seeds of Fr1 (Fig. 5d,e). During fruit development, the blue coloration was maintained from Fr1 until hardening (Fr3), but no staining was observed in the developing seeds (Fig. $5 e, f$ ). Histological sections in $\mathrm{F} 3$ anther revealed that promoter activity was localized only in the connective tissue (Fig. 5g). Sectioned Fr1 showed GUS staining in the endocarp, especially in the area where the funiculus, which was also strongly coloured, is attached to the pod wall (Fig. 5h). In more developed pods (Fr3), sections revealed high activity of $p S T 4$ in the endocarp and also in the vascular bundles (Fig. 5i,j).
The pST5 did not show any activity through the flower development (Fig. $6 a$ ) nor in the early developing fruits (Fr1) (Fig. 6b). In more advanced stages of fruit development, Fr3, when the green pod presents thorns and begins to harden staining could be seen only in the vascular bundles (Fig. $6 c, d, e$ ) and this specific activity was clearly confirmed in the histological sections (Fig. 6f,g).

Developing flowers F1 showed pST6 directed GUS staining at the base of the anthers and in the ovules when the petals had not yet fully developed and the style appeared folded (Fig. 7a) and remained active in F3 (Fig. 7b) and during the formation of the fruit and early stages of seed formation (Fig. 7c-g). Transiently, during fertilization and in the early stages of fruit formation, the style showed blue coloration (Fig. $7 c-f$ ). The staining in the ovules was later observed in the seeds (Fig. 7d-f) until the fruit began to harden, once the spiral was completely developed. In Fr3, the blue colour was located in the outer vasculature (Fig. $7 g$ ) but no staining was detected in the seeds (Fig. $7 h$ ).

The cross section of the anthers from F3 allowed to determine that pST6 was lightly activated in the 

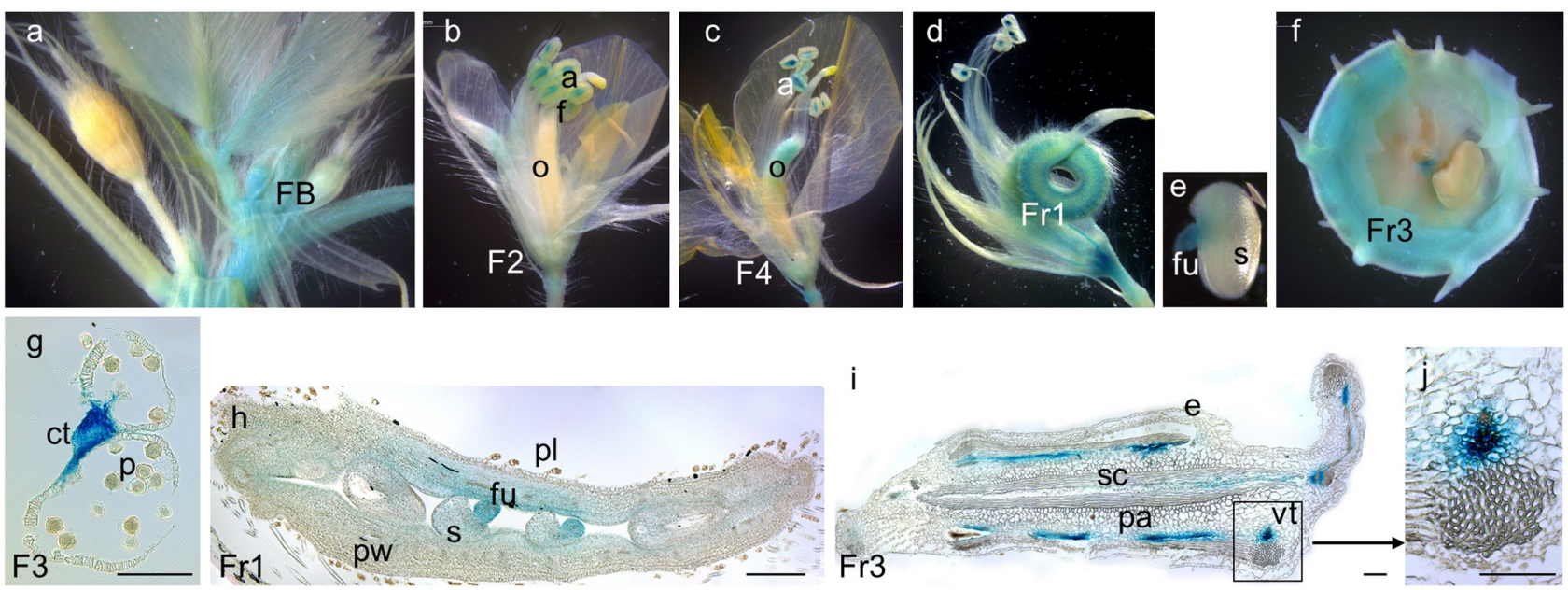

Fig. 5. Activity of Medicago truncatula pST4 in reproductive organs. Histochemical $\beta$-glucuronidase (GUS) staining during reproductive development of $M$. truncatula pST4::GUS plants, sorted according to developmental stage $(A-F)$ and histological sections after GUS staining $(G-J)$. $A$ - Floral buds (FB), $B$ - developing flower F2, $C$ - mature flower after anthesis F4, $D$ - early developing fruit Fr1, $E$ detail of developing seed in Fr1, $F$ - green pod Fr3 with seeds, $G$ - F3 anther section, $H$ - early developing fruit Fr1 section, $I$ - green pod Fr3 section, $J$ - Fr3 vascular tissue detail. The blue colour indicates the zones with GUS activity driven by $p S T 4$; a - anther, ct connective tissue, e - epidermis, f - filament, fu - funiculus, o - ovary, p - pollen, pa - parenchyma, pl - placenta, pw - pod wall, s - seed, sc - sclerenchyma, vt - vascular tissue; scale bars $=100 \mu \mathrm{m}$.
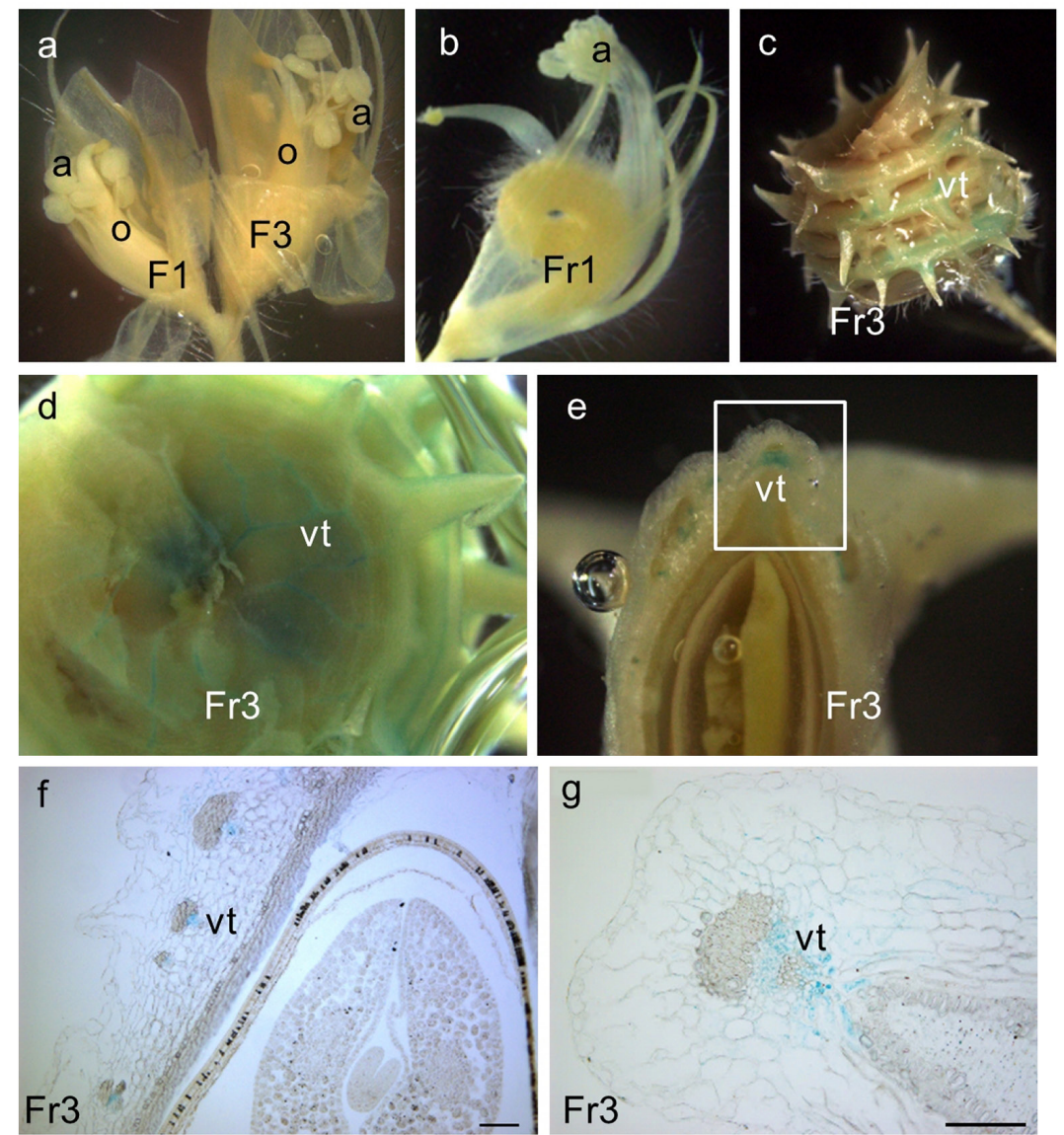

Fig. 6. Activity of Medicago truncatula pST5 in reproductive organs. Histochemical $\beta$-glucuronidase (GUS) staining during reproductive development of $M$. truncatula pST5::GUS plants, sorted according to developmental stage $(A-E)$ and histological sections after GUS staining $(F-G)$. $A$ - Developing F1 and mature F3 flowers, $B$ - early developing fruit Fr1, $C, D$ - green pod Fr3, $e$ - Fr3 transversal detail, $F$ - Fr3 section with developing seed, $G$ - Fr3 section vascular tissue detail. The blue colour indicates the zones with GUS activity driven by $p S T 5$; a - anther, o - ovary, vt - vascular tissue; scale bars $=100 \mu \mathrm{m}$. 

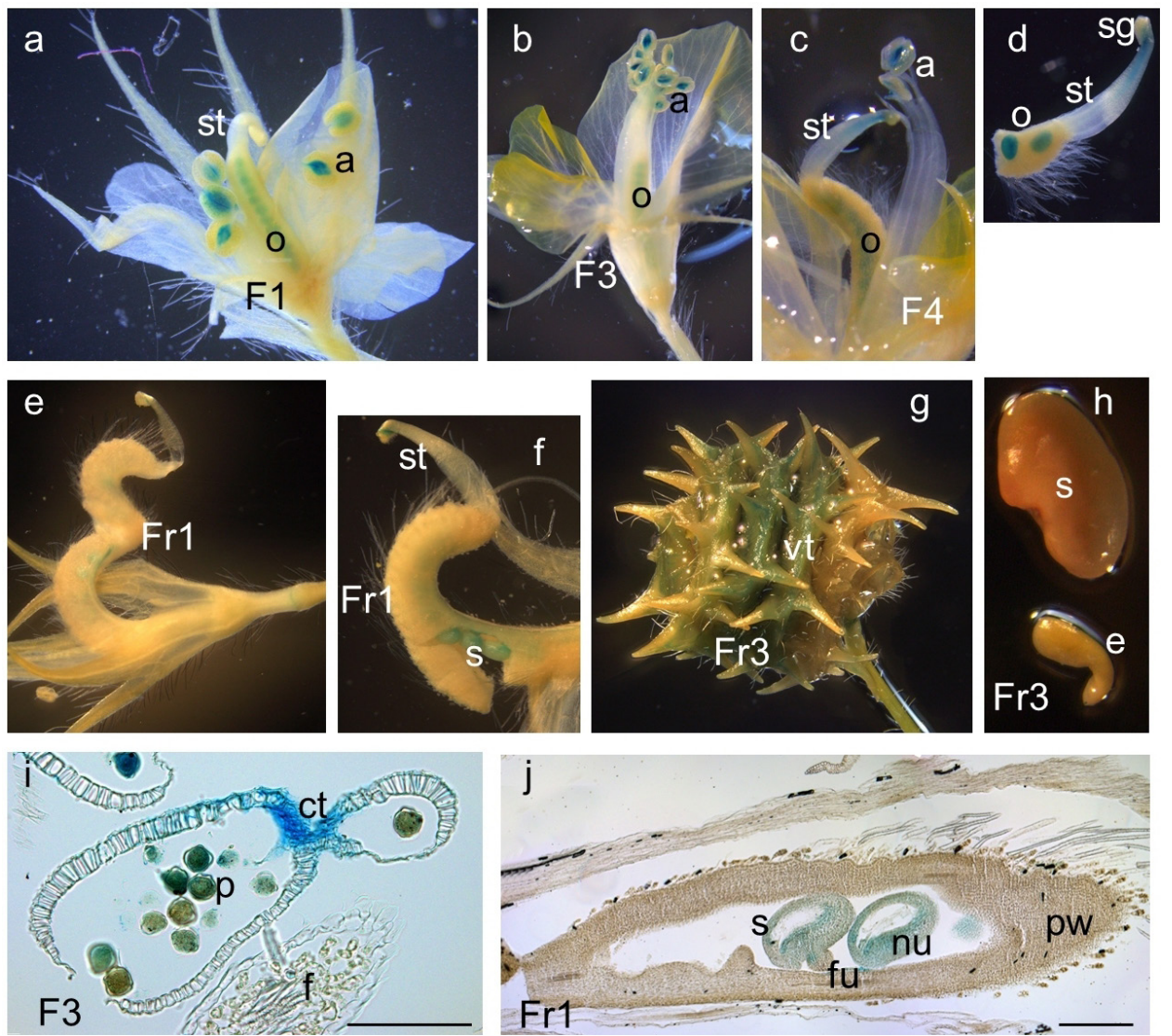

Fig. 7. Activity of Medicago truncatula pST6 in reproductive organs. Histochemical $\beta$-glucuronidase (GUS) staining during reproductive development of $M$. truncatula pST6::GUS plants, sorted according to developmental stage $(A-H)$ and histological sections after GUS staining $(I,-J)$. $A$ - Developing flower F1, $B$ - mature flower F3, $C$ - flower after anthesis F4, $D$ - detail of early developing seed in $\mathrm{F} 4$, $E, F$ - developing fruit Fr1, $G$ - green pod Fr3, $H$ - developing seed and embryo inside Fr3, $i$ - F3 anther section, $J$ - developing fruit Fr1 section. The blue colour indicates the zones with GUS activity driven by pST6; a - anther, ct - connective tissue, e - embryo, f - filament, fu - funiculus, nu - nucellus, o - ovary, p - pollen, pw - pod wall, s - seed, sg - stigma, st - style, vt - vascular tissue; scale bars $=100 \mu \mathrm{m}$ ).

parenchyma, in certain pollen grains and in some regions of the epidermis (Fig. 7i). When analysing the GUS activity in the histological sections of Fr1, it was observed that, although the pod and the funiculus did not show blue coloration, it was found in the seeds, mainly in the region of the nucellus (Fig. $7 j$ ).

\section{Discussion}

Although different ST proteins have been related to developmental processes such as early fruit morphogenesis (Fernández et al. 2007, Wechter et al. 2008), the putative function of STs during flower, fruit, and seed development remains controversial depending on the species (Williams et al. 1990, Muñoz et al. 1997, Fernández et al. 2007, Albornos et al. 2014). The fact that the ST sequences found in cDNA libraries made from mRNA isolated from seeds represent the second highest percentage after roots, and that the ST mRNAs have also been found in other reproductive organs such as flowers and fruits (Albornos et al. 2012a), point to a function of these proteins during the reproductive period of the plants.

The activity of the promoters of the six members of the $M$. truncatula ST family ( $p S T)$ during the reproductive development was previously studied in Arabidopsis thaliana transgenic plants carrying the $p S T:: G U S$ transgene and some discrepancies were found between the detected pST activity and the corresponding transcription (Albornos et al. 2017, 2018). Inevitably, any promoter outside its native context may give rise to a different activity, either due to the loss of the influence of distal elements acting on the promoter in its original location or due to the effect of foreign elements in the new region in which the transgene is inserted (Hernandez-Garcia and Finer 2014). In the aforementioned study, the different models of flower and fruit development in A. thaliana and M. truncatula could also account for the imperfect correlation between promoter activity and transcript accumulation.

To overcome the problems derived from using a heterologous plant species and to deepen in the study of the ST family during reproductive development, we have carried out the study of the activity of the $S T$ promoters in $M$. truncatula transgenic plants expressing the GUS gene driven by the six $p S T s$. In spite of the difficulty in developing these plants, which is reflected in the regeneration rates indicated in Fig. 1, it has been possible to obtain several lines of the transgenic plants containing 
any of the $6 p S T:: G U S$ transgenes. Having generated several transgenic lines allows us to compare and assess the results obtained and provides an interesting tool in the study of the ST family.

Despite the $p S T$ activity being well conserved in flowers and seeds of Arabidopsis with respect to the pattern observed in M. truncatula, the activity in the pod differs from that observed in the siliques (Albornos et al. 2017, 2018). Mainly, in the homologous system we could find the activation of every $p S T$ in the pod vasculature and sometimes in other fruit tissues where it was silent in Arabidopsis.

As a common feature to all the $p S T s$, the activity in the reproductive organs is associated with the vascular bundles, especially over the fruit formation, where there was activation in the pod vasculature at different developmental stages ( $p S T 1$ to $p S T 6$ ) (Figs. 2 to 7) and sometimes the activity was found in the funiculus ( $p S T 1$, $p S T 3$, and pST4) (Figs. 2, 3, 4). The low activity of $p S T 1$ specifically located in the vascular tissue (Fig. $2 g, h$ ) could only be detected after sectioning the tissue, while it was not evident in the whole fruit.

This association of $p S T$ with vascular cylinder relates the ST proteins with cells close to the vascular bundles, such as the transfer cells (Gunning and Pate 1969) that facilitate the arrival of nutrients. Transfer cells, among other sites, are characteristic of maternal-filial exchange zones during seed formation (Talbot et al. 2001, Royo et al. 2007). In fact, the differentiation of transfer cells has been well described in $M$. truncatula pod vascular bundles (Wang and Grusak 2005). Also in the pods, the pST3 and pST4 promoters were activated in the inner epidermis of the endocarp at the final stages of their development (Figs. 4i, 5h), where Wang and Grusak (2005) have described the formation of invaginations of the cell wall towards the cytoplasm, characteristic of the transfer cells. In legumes, these transfer cells facilitate the exchange of solutes between the seed covers and the embryo (Offler and Patrick 1993) and, in addition, the pods can function as a temporary storage of nutrients that are later transferred to the developing seed (Setia et al. 1987). In short, the activation of the $p S T$ in the proximity of the vascular bundles, especially in the pod (Figs. 2 to 7 ) indicates their relationship with transfer cells that enable the arrival of nutrients to the new organs, which would support a function of STs in the storage and/or mobilization of reserves as has previously been indicated (Albornos et al. 2014).

In $M$. truncatula, it has been established that $S T$ transcription and $p S T$ activity are usually much higher in roots than in shoots (Albornos et al. 2017, 2018). It is known that developing roots are a sink for $\mathrm{P}$ and photoassimilated carbon which are transported from the shoot to the root (Marschner et al. 1996, Wissuwa et al. 2005). Later in plant development, in the reproductive phase, flowers and fruits become the major sink organs and therefore there is a shift in the nutrient allocations (Peng and Li 2005). Thus, the presence of ST transcript and proteins in flowers and fruits in different species would support a role in nutrient transport and exchange during fruit development. In pea, PsaST3 was described as a pod specific mRNA (Williams et al. 1990) and in chickpea, the protein CarST1 was immunolocalized to green pod endocarp (Albornos et al. 2014). Also, research on the development of fleshy fruits, such as grapes (Waters et al. 2005, Fernández et al. 2007) and watermelon (Levi et al. 2006, Wechter et al. 2008) showed that ST proteins could be involved in early fruit morphogenesis, as transcripts accumulate before maturation (Fernández et al. 2007) and decline in the mature fruit (Wechter et al. 2008).

In addition to these common characteristics found for the $p S T$ activities during the reproductive development, our present results reinforce and sometimes supplement the functions previously established for the three functional group of ST proteins described in M. truncatula.

The pST1 activity in $M$. truncatula, which was specifically located in the pollen of flowers in stages F2 to F4 (Fig. 2b,c,f) as well as in the funiculus and in the seed hilum of Fr3 fruit (Fig. $2 d-e$ ), coincides with the previously reported activity in Arabidopsis (Albornos et al. 2017) and supports the proposed function for ST1 in the transport and assimilation of nutrients (Albornos et al. 2017), as the funiculus provides a way for nutrients to move from the mother plant (Schulz and Jensen 1971, Meinke 1994). The fact that the $S T 1$ gene is differentially regulated upon changes in the nutritional status of the plant is supported by several data. Firstly, the transcript accumulation in nitrogen and phosphate starvation (Albornos et al. 2017); secondly the pST1 showing CREs related to nitrogen metabolism and phosphate transporters (Albornos et al. 2017); and finally, the publicly available gene expression experiments using microarrays that show an increase in ST1 transcription under limited nitrogen availability (MtGEA, Beneditto et al. 2008).

This ST1 function was reinforced by the detection of $p S T 1$ activity in the vascular bundles after the histological sectioning of Fr3 fruits (Fig. $2 g, h$ ), and relates it to the reserve storage and mobilization in the reproductive phase, consistent with the reported accumulation of ST1 transcripts in developing fruits and seeds (Albornos et al. 2017). In this sense, it is worth mentioning that the chickpea protein CarST1, belonging to the same structural group than ST1, accumulates in cotyledons of dry seeds and decreases during germination until finally disappearing in the exhausted cotyledons (Albornos et al. 2014) resembling the behaviour of seed storage protein.

The $p S T 2$ and $p S T 3$ are only activated in early stages of seed development in Fr1 and Fr2 (Fig. 3c, Fig. 4d,e), but no activity is found in later stage of seed formation (Fr3; Fig. 3e, Fig. 4g). However, ST2 and ST3 have been related to water stress and to physiologically controlled desiccation processes such as the seed maturation (Albornos et al. 2017), based on the high amount of transcripts found at the final stage of seed formation and the high amount of CREs related to the gene expression in seeds found in their promoter sequences (Albornos et al. 2017). The inconsistency between the strong transcript accumulation in the green seeds (Albornos et al. 2017) and the disappearance of the $p S T 2$ and $p S T 3$ activity in seeds at the final stages of their formation (Figs. 3 and 4) had already been observed when the pST activity was studied 
in Arabidopsis (Albornos et al. 2017), which validates our previous results, and indicates that this discrepancy is not due to the different type of fruit and seed development in both species. However, our present results in M. truncatula allow us to have a clearer picture of the $p S T$ activity pattern and to explain these differences considering the specificity of the $p S T$ activity in the different parts of the ovules and pods during the seed formation. The high $p S T 3$ activity that can be appreciated in the placenta, funiculus, and the external part of the developing seed points to a high transcription rate of ST3 gene at these early stages (Fig. 4j). These high amount of transcripts could accumulate untranslated in the seeds while their development is progressing and would remain in the integuments of the seeds when they become green and mature, which can explain why mRNA can be detected (Albornos et al. 2017) even though at this final stage there is not pST3 activity in green seeds, except for some staining in the funiculus (Fig. $4 k$ ). This can also be observed for pST2 (Fig. 3f,g). A high representation of ST3 transcript and an increase of these transcripts in seed at $36 \mathrm{~d}$ after pollination was also reported in expression profiles from platform Genevestigator (Hruz et al. 2008) and MtGEA (Beneditto et al. 2008). A similar behaviour has been observed for $S T 2$ gene regarding promoter activity (Fig. 3f,g) and transcript accumulation (Albornos et al. 2017) indicating that it could be regulated in the same way.

Regarding to the third functional group of ST proteins (ST4, ST5, and ST6), the promoter activity of each one was different along reproductive development, except for its above-mentioned presence in vascular bundles. It is worth noting the high activity of pST4 in floral buds (Fig. $5 a$ ), the only one which shows high activity at this stage of the floral development, matching the accumulation of transcripts found at this stage in the flowers (Albornos et al. 2018). The pST4 activity in floral buds was also detected in Arabidopsis transgenic plants (Albornos et al. 2018), although in these species no other activity was found during the reproductive stage. However, in M. truncatula transgenic plants the activity of this promoter is also detected during flower and fruit development (Fig. 5) despite the absence of mRNA accumulation in these stages (Albornos et al. 2018) which points to the existence of extra control of the transcription beyond the promoter sequence.

No pST5 activity was detected throughout the reproductive phase (Fig. 6), with the exception of the already mentioned faint activity in the fruit vascular bundles, which coincides with the absence of transcripts in the reproductive period and resolves the discrepancy found in Arabidopsis transgenic plants, where pST5 activity was detected in developing anthers, at the base of the youngest siliques and in the seed chalazal tissue (Albornos et al. 2018), which could indicate a mis-regulation of this promoter in different plant species.

The pST6 was active along flower development (Fig. 7), as it happened in Arabidopsis transgenic plants and coinciding with the pattern of transcripts accumulation (Albornos et al. 2018). The detection of GUS activity in the seeds of young fruits, mainly in the nucellar region, presents a similar pattern than the described for $p S T 2$ and $p S T 3$, and again can explain the discrepancy between the low activity of its promoter and the high levels of transcripts found in the green seeds (Albornos et al. 2018). It is remarkable that these three proteins (ST2, ST3 and ST6) are the only ones that present a double subcellular location, both in the cell wall and in the cytoplasm (Albornos et al. 2017, 2018).

This third group of M. truncatula STs (ST4, ST5, and ST6) has been linked to the interactions with other organisms, and their transcripts are specifically and differentially up-regulated by MeJA, ET, and SA, plant regulators involved in the plant defence. Also, in publicly available gene expression experiments using microarrays we have found that ST6 is upregulated in roots infected with Ralstonia solanacearum in two different genotypes (Beneditto et al. 2008). Although flowers are attacked by herbivores and pathogens, floral defence as compared with leaf defence, is rarely studied. Some literature describes the accumulation of defensive proteins in flowers but the signalling that regulate these defences are unknown. Thus, the pathogenesis related protein PR-1 has been reported to be accumulated constitutively during the normal development of the tobacco flower, even in the absence of microbial attack or other stresses (Lotan et al. 1989), although the specific function of this protein in flowers is unknown. Damle et al. (2005) indicated a higher accumulation of proteinase inhibitors in tomato flowers than in leaves as a possible way to counteract the feeding preference of Helicoverpa armigera, and more recently, Li et al. (2017) reported that in Nicotiana attenuata, early flower stages constitutively accumulate large amounts of tripsin proteinase inhibitors, and that a flower-specific sector of JA signalling regulates these constitutively expressed floral defences. In our study, the activation of pST4, pST5 and pST6 in flowers (Fig. 5, 6, 7), considering the strong induction of the ST4, ST5 and ST6 transcripts caused by the hormones involved in plant defence (Albornos et al. 2018), could mean that these ST proteins also participate in the defence of the reproductive organs, according to the proposed function for this ST group, although more work is necessary to establish this point.

\section{References}

Albornos, L., Cabrera, J., Hernández-Nistal, J., Martín, I., Labrador, E., Dopico, B.: Organ accumulation and subcellular location of Cicer arietinum ST1 protein. - Plant Sci. 224: 44$53,2014$.

Albornos, L., Martín, I., Hernández-Nistal, J., Labrador, E., Dopico, B.: Three members of Medicago truncatula ST family (MtST4, MtST5 and MtST6) are specifically induced by hormones involved in biotic interactions. - Plant Physiol. Biochem. 127: 496-505, 2018.

Albornos, L., Martín, I., Iglesias, R., Jiménez, T., Labrador, E., Dopico, B.: ST proteins, a new family of plant tandem repeat proteins with a DUF2775 domain mainly found in Fabaceae and Asteraceae. - BMC Plant Biol. 12: 207, 2012a.

Albornos, L., Martín, I., Labrador, E., Dopico, B.: Three members of Medicago truncatula ST family are ubiquitous 
during development and modulated by nutritional status (MtST1) and dehydration (MtST2 and MtST3). - BMC Plant Biol. 17:117, 2017.

Albornos, L., Martín, I., Pérez, P., Marcos, R., Dopico, B., Labrador, E.: Promoter activities of genes encoding $\beta$-galactosidases from Arabidopsis al subfamily. - Plant Physiol. Biochem. 60: 223-232, 2012 b.

Barker, D.G., Faff, T., Moreau, D., Groves, E., Ruffel, S., Lepetit, M., Whitehand, S., Maillet, F., Nair, R.M., Journet, E.: Growing M. truncatula: choice of substrates and growth conditions. In: Mathesius, U., Journet, E., Sumner, L. (ed.): The Medicago truncatula Handbook. Pp. 1-26. The Samuel Roberts Noble Foundation, Oklahoma 2006.

Benedito, V.A., Torres-Jerez, I., Murray, J.D., Andriankaja, A., Allen, S.N., Kakar, K., Wandrey, M., Verdier, J., Zuber, H., Ott, T., Moreau, S., Niebel, A., Frickey, T., Weiller, G.W., He, J.W., Dai, X., Zhao, P.X., Tang, Y., Udvardi, M.K.: A gene expression atlas of the model legume Medicago truncatula. Plant J. 55: 504-513, 2008.

Chabaud, M., De Carvalho-Niebel, F., Barker, D.: Efficient transformation of Medicago truncatula cv. Jemalong using the hypervirulent Agrobacterium tumefaciens strain AGL1. Plant Cell Rep. 22: 46-51, 2003.

Damle, M.S., Giri, A.P., Sainani, M.N., Gupta, V.S.: Higher accumulation of proteinase inhibitors in flowers than leaves and fruits as a possible basis for differential feeding preference of Helicoverpa armigera on tomato (Lycopersicon esculentum Mill, cv. Dhanashree). - Phytochemistry 66: 26592667, 2005.

De Vries, S.C., De Vos, W.M., De Harmsen, M.C., Wessels, J.G.H.: A shoot-specific mRNA from pea: nucleotide sequence and regulation as compared to light-induced mRNAs. - Plant mol. Biol. 4: 95-102, 1985.

Doyle, J.J., Doyle. J.L.: A rapid DNA isolation procedure for small quantities of fresh leaf tissue. - Phytochem. Bull. 19: 11-15, 1987.

Fernández, L., Torregrosa, L., Terrier, N., Sreekantan, L., Grimplet, J., Davies, C., Thomas, M.R., Romieu, C., Ageorge, A.: Identification of genes associated with flesh morphogenesis during grapevine fruit development. - Plant mol. Biol. 63: 307-323, 2007.

Germain, H., Lachance, D., Pelletier, G., Fossdal, C.G., Solheim, H., Séguin, A.: The expression pattern of the Picea glauca Defensin 1 promoter is maintained in Arabidopsis thaliana, indicating the conservation of signalling pathways between angiosperms and gymnosperms. - J. exp. Bot. 63: 785-795, 2012.

Gaude, N., Bortfeld, S., Duensing, N., Lohse, M., Krajinski, F.: Arbuscule-containing and non-colonized cortical cells of mycorrhizal roots undergo extensive and specific reprogramming during arbuscular mycorrhizal development. - Plant J. 69: 510-528, 2012.

Gunning, B., Pate, J.: "Transfer cells" plant cells with wall ingrowths, specialized in relation to short distance transport of solutes - their occurrence, structure, and development. Protoplasma 68: 107-133, 1969.

Hernandez-Garcia, C.M., Finer, J.J.: Identification and validation of promoters and cis-acting regulatory elements. - Plant Sci. 217-218: 109-119, 2014.

Hernández-Nistal, J., Labrador, E., Martín, I., Jiménez, T., Dopico, B.: Transcriptional profiling of cell wall protein genes in chickpea embryonic axes during germination and growth. - Plant Physiol. Biochem. 44: 684-692, 2006.

Hernández-Nistal, J., Martín, I., Esteban, R., Dopico, B., Labrador, E.: Abscisic acid delays chickpea germination by inhibiting water uptake and downregulating genes encoding cell wall remodelling proteins. - Plant Growth Regul. 61: 175$183,2010$.

Hruz, T., Laule, O., Szabo, G., Wessendorp, F., Bleuler, S., Oertle, L., Widmayer, P., Gruissem, W., Zimmermann, P.: Genevestigator V3: a reference expression database for the meta-analysis of transcriptomes. - Adv. Bioinformatics 2008: 420747, 2008.

Levi, A., Davis, A., Hernandez, A., Wechter, P., Thimmapuram, J., Trebitsh, T., Tadmor, Y., Katzir, N., Portnoy, V., King, S.: Genes expressed during the development and ripening of watermelon fruit. - Plant Cell Rep. 25: 1233-1245, 2006.

Li, R., Wang, M., Wang, Y., Schuman, M.C., Weinhold, A., Schäfer, M., Jiménez-Alemán, G.H., Barthel, A., Baldwin, I.T.: Flower-specific jasmonate signaling regulates constitutive floral defenses in wild tobacco. - Proc. nat. Acad. Sci. USA 114: E7205-E7214, 2017.

Liu, J., Maldonado-Mendoza, I., Lopez-Meyer, M., Cheung, F., Town, C.D., Harrison, M.J.: Arbuscular mycorrhizal symbiosis is accompanied by local and systemic alterations in gene expression and an increase in disease resistance in the shoots. - Plant J. 50: 529-544, 2007.

Lotan, T., Ori, N., Fluhr, R.: Pathogenesis-related proteins are developmentally regulated in tobacco flowers. - Plant Cell 1: 881-887, 1989.

Marschner, H., Kirkby, E.A., Cakmak, I.: Effect of mineral nutritional status on shoot-root partitioning of photoassimilates and cycling of mineral nutrients. - J. exp. Bot. 47: 1255-1263, 1996.

Meinke, D.W.: Seed development in Arabidopsis thaliana. - In: Meyerowitz, E.M., Somerville, C.R. (ed.): Arabidopsis. Pp. 253-295. Cold Spring Harbor Laboratory Press, Cold Spring Harbor 1994.

Muñoz, F.J., Dopico, B., Labrador, E.: Two growth-related organ-specific cDNAs from Cicer arietinum epicotyls. - Plant mol. Biol. 35: 433-442, 1997.

Offler, C.E., Patrick, J.W.: Pathway of photosynthate transfer in the developing seed of Vicia faba L: a structural assessment of the role of transfer cells in unloading from the seed coat. - J. exp. Bot. 44: 711-724, 1993.

Peng, Z., Li, C.: Transport and partitioning of phosphorus in wheat as affected by $\mathrm{P}$ withdrawal during flag-leaf expansion. - Plant Soil 268: 1-11, 2005.

Royo, J., Gómez, E., Hueros, G.: Transfer cells. - In: Olsen O.A. (ed.): Endosperm. Pp. 73-89. Springer, Dordrecht 2007.

Schenk, R.U., Hildebrandt, A.C.: Medium and techniques for induction and growth of monocotyledonous and dicotyledonous plant cell cultures. - Can. J. Bot. 50: 199-204, 1972.

Schulz, P., Jensen, W.A.: Capsella embryogenesis: the chalazal proliferating tissue. - J. Cell Sci. 8: 201-227, 1971.

Setia, R.C., Setia, N., Malik, C.P.: The pod wall structure and function in relation to seed development in some legumes. Phyton 27: 205-220, 1987.

Talbot, M.J., Franceschi, V.R., McCurdy, D.W., Offler, C.E.: Wall in growth architecture in epidermal transfer cell of Vicia faba cotyledons. - Protoplasma 215: 191-203, 2001.

Terzaghi, W.B., Cashmore, A.R.: Light-regulated transcription. Annu. Rev. Plant Physiol. Plant mol. Biol. 46: 445-474, 1995.

Wang, H.L., Grusak, M.A.: Structure and development of Medicago truncatula pod wall and seed coat. - Ann. Bot. 95: 737-747, 2005.

Waters, D. L., Holton, T. A., Ablett, E. M., Lee, L. S., Henry, R. J.: cDNA microarray analysis of developing grape (Vitis vinifera cv. Shiraz) berry skin. - Funct. Int. Gen. 5: 40-58, 2005.

Wechter, W.P., Levi, A., Harris, K.R., Davis, A.R., Fei, Z., Katzir, 
N., Giovannoni, J.J., Salman-Minkov, A., Hernandez, A., Thimmapuram, J., Tadmor, Y., Portnoy, V., Trebitsh, T.: Gene expression in developing watermelon fruit. - BMC Genomics 9: 275,2008

Williams, M.E., Mundy, J., Kay, S.A., Chua, N.-H.: Differential expression of two related organ-specific genes in pea. - Plant mol. Biol. 14: 765-774, 1990.

Wissuwa, M., Gamat, G., Ismail, A.M.: Is root growth under phosphorus deficiency affected by source or sink limitations? - J. exp. Bot. 56: 1943-1950, 2005. 